(Mitteilung aus der Jenaer Augenklinik und der Zeissschen Werkstätte.)

\title{
Über eine Methode zur subjektiven Prüfung von Brillenwirkungen.
}

\author{
Von \\ M. v. Rohr und W. Stock.
}

Mit 7 Figuren im Text.

Bei der Beurteilung der Wirkung von Brillengläsern war man bis jetzt ausschliesslich auf das Urteil der Patienten angewiesen, und das war um so unangenehmer, je mehr Arbeit auf die als Brillen dienenden Konstruktionen verwandt worden war. Es konnte sehr wohl auch beim besten Willen des Patienten der Fall eintreten, dass die Brillenkonstruktion auf Grund einer individuellen Besonderheit, etwa seines Augenhintergrundes oder seines Muskelapparats, verworfen wurde. Ein solcher Misserfolg entmutigte in gleicher Weise den experimentierenden Arzt und den Konstrukteur, obwohl sich beide die Möglichkeit einer derartigen Erklärung vor Augen halten mussten. Häufiger allerdings trat der Fall ein, dass dem Patienten die Geduld ausging oder dass ihm - sei es im Hinblick auf eine mögliche Rentenverminderung, sei es aus Furcht, durch das Tragen einer auffallenden Brillenkonstruktion an Schönheit einzubüssen - überhaupt der gate Wille fehlte. Immerhin bot aber auch eine solche Erklärung eines negativen Ausfalls nur einen schwachen Trost. Der Konstrukteur konnte in allen derartigen Fällen nur darauf hinweisen, dass das Linsensystem den vorgeschriebenen Bedingungen genüge - dazu konnte er sich auf seine Rechnungen und gegebenenfalls auf photographische Probeaufnahmen berufen - aber eine solche Zurückweisung eines Fehlerverdachts konnte nichts Positives beweisen, konnte unmöglich für die Anwendbarkeit der Konstruktion irgendwie entscheidend sein.

Es liegt eben in der eigentümlichen Anlage des Menschenauges begründet, dass beim Einzelauge das Wertungsverhältnis zwischen dem 
Gesichtsfeld beim ruhenden und dem Blickfeld beim bewegten Auge wahrscheinlich mit der Art der Objekte schwankt und daher nicht leicht in eine bestimmte Formel gefasst werden kann; ferner kann anch die beste Kontrolle durch die Photographie nur unvollkommen und schwerfällig den leichten Bewegungen des blickenden Auges folgen. Kommt man nun aber gar zum beidäugigen Sehen durch Brillen, so ist man heute noch weit davon entfernt, die Bedingungen zu kennen, unter denen es statthaben kann, und unter denen es unmöglich wird. Um nur einiges heranszugreifen, sei daran erinnert, dass uns vorderhand alle Angaben darüber fehlen, welche Höhenfehler von dem Durchschnitt der Beobachter noch überwunden werden können, und dass es ähnlich mit unserer Kenntnis der Fähigkeit steht, womit Bilder verschiedener Grösse etwa von Anisometropen noch zu einem einheitlichen stereoskopischen Eindruck vereinigt werden können.

Eine Reihe dieser Schwierigkeiten der Beurteilung würde in demselben Augenblicke schwinden, wo es gelänge, die verschiedenen Brillenkonstruktionen durch einen sachverständigen Beobachter mit physiologisch-psychologischer Schulung prüfen zu lassen. Man sieht ohne weiteres ein, dass diese Forderung darauf hinaus kommt, die Refraktion des optischen Systems im Auge nach Belieben zu ändern, ohne doch die charakteristische Bewegung des ganzen Systems um den Augendrehpunkt zu beeinflussen. Wenn aber das Brillenglas an der Bewegung des Auges teilnehmen soll, so bleibt nichts weiter übrig, als zu der Konstruktion zu greifen, die A. E. Ficks) im Jahre 1888 unter dem Namen der Kontaktbrille veröffentlichte und hauptsächlich zur Abhilfe der Folgen von irregulärem Astigmatismus bestimmte.

Die nähere Geschichte der Kontaktbrillen scheint nicht allgemein bekannt zu sein, sie ist aber so interessant, dass es sich wohl lohnt, sie hier mit einigen Worten mitzuteilen.

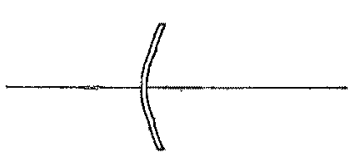

A. E. Fick gibt auf S. 285 seiner $\mathrm{Ab}$ handlung die folgende Beschreibung seiner Erfindung (Fig. 1). „Eine Glascornea von $8 \mathrm{~mm}$ Krümmungsradius sitzt mit einer Basis von Fig. 1. Skizze für das Fi cksehe Kontaktglas in etwa $2 / 37 \mathrm{~mm}$ Radius auf der Glassclera; die letztere natürlicher Grösse. $\quad$ ist $3 \mathrm{~mm}$ breit und entspricht einer Kugel von $15 \mathrm{~mm}$ Krümmungsradius; die Glascornea ist parallelwandig, anssen und innen geschliffen und poliert; desgleichen ist der freie Rand der

1) A. E. Fick, Eine Contactbrille. Arch. f. Augenhoilk. Bd. XVIII. S. 279 bis 289. 1888. (Sept. 1887.) 
Glassclera geschliffen und poliert; Gewicht einer Contactbrille etwa $0,5 \mathrm{~g}$." Von Interesse ist es, dass sich um dieselbe Zeit der Pariser Ophthalmologe E. Kalt ${ }^{1}$ ) unabhängig mit demselben Problem beschäftigte, dass ihm aber bei der Publikation A. E. Fick zuvorgekommen war. Das gleiche Korrektionsmittel wurde dann einige Jahre später - 1892 - von D. E. Sulzer ${ }^{2}$ ) wieder aufgenommen, der in seiner Veröffentlichung auf einen Irrtum A. E. Ficks hinwies. Dessen Kontaktgläser seien nicht geschliffen gewesen, wie er angenommen habe, sondern nur geblasen. Er selber habe gute Kontaktgläser - wie aus A. E. Ficks Antwort hervorgeht, aus einem Stück geschliffene - von Benzoni aus Genf und von der Firma Berthier aus Paris herstellen lassen. Aus A. E. Ficks3) Antwort kann man weiter entnehmen, dass seine ersten, durch die Vermittlung von $\mathbf{E}$. A bbe bezogenen Gläser in der Tat nur geblasen waren, und dass er später allein durch eine Gefälligkeit der Berliner Firma O. Himmler in den Besitz geschliffener Kontaktbrillen gekommen sei. Seine Versuche scheinen besonders an dem hohen Preise der Einzelgläser gescheitert zu sein, der den französischen (von $30 \mathrm{Fr}$.) um $56 \%$ übertraf. Man wird heute wohl an dem Preise, der bei tadelloser Ausführung sehr hoch ansfallen muss, ein wichtiges Hindernis dafür sehen, dass sich dieses so scharfsinnig erdachte Korrektionsmittel nicht besser eingeführt hat.

Wie man aus der Beschreibung sieht, handelt es sich dabei um eine stark durchgebogene Linse, deren Innenfläche die Scheitelkrümmung der vorderen Hornhautfläche erhält, so dass sie sich eng an sie anlegt. Verwendet man dieses Mittel zur Korrektion von Achsenametropien, so liegt es auf der Hand, dass man dann durch Einführung einer geeigneten Krümmungsametropie den emmetropischen Zustand erreicht. An der Augendrehung und somit an den Blickwinkeln wird nichts geändert, dagegen wird die Brechkraft des optischen Systems im korrigierten Auge einen abweichenden Wert erhalten, und auch die Kardinalpunkte werden eine Verlagerung erfahren.

1) Traitement optique du kératocône. Ann. d'ocul. XCIX. p. 293. 1888 (Juniheft).

2) Sulzer, D. E., La correction optique du kératocône, de l'astigmatisme irrégulier et de l'astigmatisme cicatriciel. $2+$. Bull. et Mém. Soc. Franę. d'Opht. X. p. 118-120. 1892 und fast ganz wörtlich: Ann. d'ocul. CVII. p. 321-328. 1892.

9) Fick, A. E., Einige Bemerkungen über die Contactbrille (25. VII. 92). Klin. Monatsb1. f. Augenheilk. Bd. XXX. S. 306-310. 1892. 
Die Formeln für die Ermittlung dieser Änderungen würden sich - wenn man etwa von dem Gullstrandschen als dem heute vollkommensten Augenschema ausginge - obne Schwierigkeit hinschreiben lassen, doch ist nicht anzunehmen, dass sich die Mebrzahl der Leser durch eine solche Ableitung befriedigt erklärte. Wir beschränken uns daher auf eine Mitteilung der Resultate der Rechnung in der Form einer graphischen Darstellung und glauben, durch eine solche übersichtliche Vorfuhrung der stetigen Lagenänderung aller wichtigen Punkte immerhin einen nützlichen Beitrag zur Kenntnis der korrigierenden Fickschen Kontaktbrille geliefert zu haben. Als Schema benutzen wir die Anlage, die einer ${ }^{1}$ ) von uns im vorigen Jahre für die Darstellung der optischen Konstanten achsenametropischer Augen benutzt hat, deren korrigierende dïnne Brillengläser ganz oder doch angenähert im vorderen Augenbrennpunkt $\mathbf{F}$ angebracht waren. Aber auch hier ist darauf hinzuweisen, dass diese Überlegung nur für die Verhältnisse im paraxialen Raum gilt, ohne weiteres also nur Aufschluss gibt über die Grösse des Bildes auf der Netzhaut. Hinsichtlich der Lage dieses Bildes erhält man nur den Gaussschen Grenzwert, dem sich der tatsächliche Bildort um so mehr nähert, je kleiner der Durchmesser der Pupille ist, und zwar deshalb, weil die sphärische Aberration im optischen System des Auges bei dieser Rechnung nicht berücksichtigt worden ist.

Trotz dieser Beschränkung der strengen Gültigkeit wird aber immerhin diese erste Stufe der Annäherung ein gewisses Interesse bieten, da sie für eine Reihe von Kontaktbrillen systematisch durchgefuihrt wurde.

Die hier in der Fig. 2 gegebene Darstellung entspricht den drei an der citierten Stelle als Fig. 4, 5 und 6 mitgeteilten Kurvensystemen hinsichtlich des berücksichtigten Gebietes. Es war indessen nötig, den rechten Teil, der die Vergrösserung des Netzhautbildes gegenüber seiner Grösse im normalen Auge darstellt, als Fig. 3 von dem vorangehenden Teile abzutrennen, denn sonst würde sich eine zu grosse Breite der Darstellung ergeben haben. Da es sich bei der vorliegenden Fickschen Kontaktbrille immer um ein korrigierendes Glas handelt, so kann man erwarten, dass sich die Darstellung am meisten der oben citierten Fig. 5 annähern wird, für die das gewöhnliche Brillenglas zwischen dem vorderen Brennpunkt und dem vorderen

1) y. Rohr, M., Die Brille als optisches Instrument. Graefe-SaemischHesssches Handb. d, ges. Augenheilk. 2. Anf. Siehe S. 14-16. 
Hauptpunkt des unbewaffneten Auges angebracht worden war. In dieser Erwartung sieht man sich auch nicht getäuscht, denn es ist für die neue Kurvendarstellung charakteristisch, dass der Abstand des vorderen Brennpunkts $\mathbf{F}$ des korrigierten Systems vom Hornhautscheitel in dem gleichen Sinne variiert, wie die Achsenlänge, während sich die Hauptpunkte des kombinierten Systems nur sehr unwesentlich gegen die Lage der Augenhauptpunkte verschoben haben. Dieser Umstand soll weiterhin noch näher besprochen werden. Man sieht also, dass bei dem für die Kontaktbrille gültigen Schema die Kurve FF aus

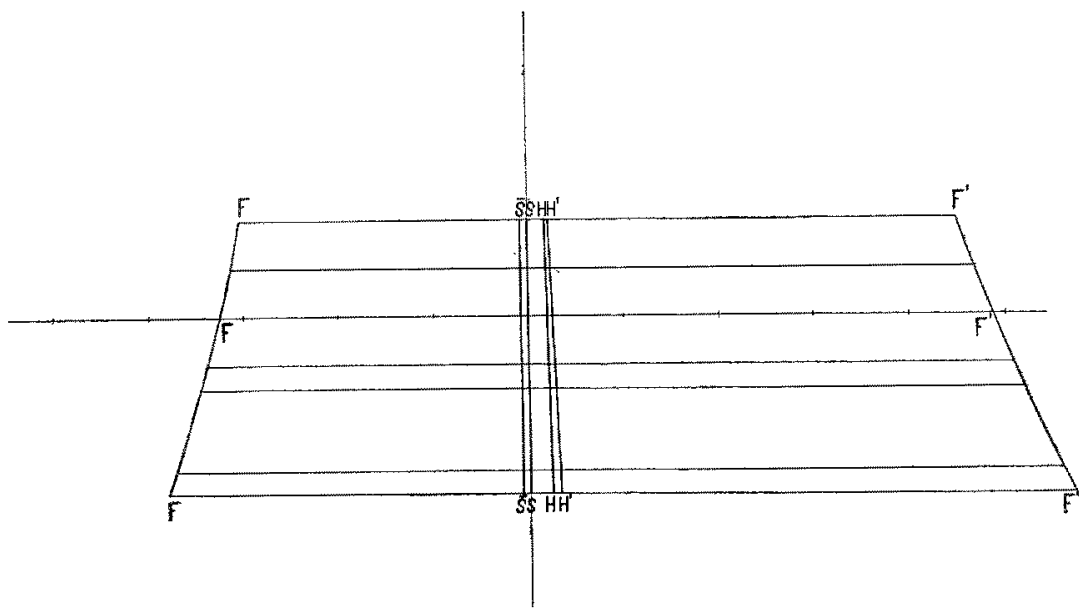

Fig. 2. Ein Diagramm für die Lage der Kardinalpunkte des aus Vollauge und Fickschem Kontaktglas gebildeten Systems für Ametropien zwischen +5 und $-10 \mathrm{dptr}$

FE vorderer, $\mathbf{F}^{\prime} \mathbf{F}^{\prime}$ hinterer Brennpunkt. HH worderer, $\mathbf{H}^{\prime} \mathbf{H}^{\prime}$ hinterer Hauptpunkt. SS Hornhautscheitel. $\overline{\mathrm{SS}}$ Scheitel des Kontaktglases.

der nur schwach nach unten und aussen geneigten Lage der citierten Fig. 5 in eine Stellung gelangt, die sehr entschieden in diesem Sinne geneigt ist. Hält man das mit dem Umstande zusammen, dass die Hauptpunkte des kombinierten Systems nur sehr geringe Lagenänderungen erfahren haben, so folgt aus der vorliegenden Darstellung, dass die Korrektion der Achsenametropie in dem Falle der Fickschen Kontaktbrille fast ausschliesslich durch die Verkürzung oder Verlängerung der Brennweite (das ist eine Änderung der Brechlkraft) erfolgt. Dagegen spielt hier so gut wie keine Rolle die Verschiebung des hinteren Hauptpunkts, die beispielsweise in der Fig. 4 bei der ideal, das ist im vorderen Augenbrennpunkt angeordneten dünnen Brille die Ausgleichung allein, in den Fig. 5 und 6 bei den zu nah oder zu fern stehenden dünnen Brillen doch wenigstens zum Teil besorgte.

४. Graffe's Arehiv für Ophthalmologie. LXXXIII. 1. 
Dem entspricht natürlich auch unsere Darstellung in Fig. 3 der Vergrösserung des Netzhautbildes. Zwar der Charakter dieser Kurve Verkleinerung für stärkere Hyperopien, Vergrösserung für stärkere Myopien - stimmt mit dem der citierten Fig. 5 überein, aber die Beträge dieser Massstabsänderung erreichen ungefähr die doppelte Grösse der unter jenen Umständen ermittelten Werte.

Was nun die Lagenänderung der

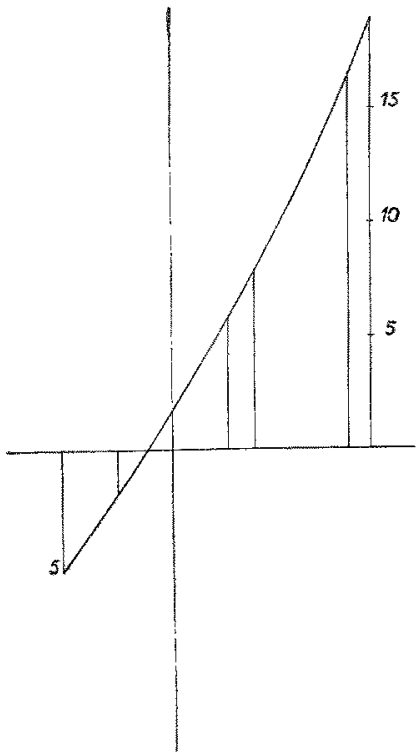

Fig. 3. Schema für die Vergrösserung des Netzhantbildes für diezwischen $+5 \mathrm{u},-10 \mathrm{dptr}$ liegenden KontaktgläserinDifferenzen gegen die Einheit. Den obigen Grenzen entsprechen etwa dieVergrösserungen von 0,95 and 1,19 . Hauptpunkte angeht, so ist darauf hinzuweisen, dass die Kontaktlinsen, die für dieses Beispiel mit einer gerade noch ausführbaren Mitteldicke von $0,4 \mathrm{~mm}$ angenommen worden sind, nicht im Sinne der rechnenden Optik als dünne Linsen gelten können. Dazu sind die Linsenkrümmungen zu stark, und das wird auch ohne Vorführung eines Musterbeispiels verständlich, wenn man beachtet, dass bei einem Kontaktglas aus Kron vom Brechungsexponenten

$$
n_{0}=1,52
$$

der zerstreuenden Fläche mit

eine Brechkraft von

$$
r_{2}=7,7 \mathrm{~mm}
$$

$$
D_{1}^{\prime \prime}=-67,5 \mathrm{dptr}
$$

zukommt.

Aus diesem Grunde ist hier auch stets die Dicke von $0,4 \mathrm{~mm}$ in der Rechnung berücksichtigt worden, und es hat sich dabei gezeigt, dass die Hauptpunkte einer solchen Fickschen Kontalktbrille keineswegs angenähert mit der Lage ihrer beiden Scheitel übereinstimmen. Namentlich bei den Kontaktbrillen geringer Brechkraft ergeben sich für die Brillenhauptpunkte Scheitelabstände von $7 \mathrm{~mm}$ und mehr. Man erkennt leicht, dass man unter Berücksichtigung der Hauptpunktabstände des optischen Systems im schematischen Vollauge exakte Werte für die Kardinalpunkte des mit einer Kontaktbrille bewaffneten Vollauges nicht erwarten kann, wenn man das endliche, aus jenen Abständen folgende Interstitium

$$
\delta=H_{1}^{\prime} \mathbf{H}
$$

einfach vernachlässigt. Wie bereits erwähnt, hat die Ficksche Kor- 
rektionsmethode zur Folge, dass sich die Hauptpunkte des optischen Systems von ihrer Lage beim schematischen Auge ausgehend etwas nach vorn verschieben, doch würde sich diese Verlagerung wegen der ziemlich geringen Versuchsgenauigkeit bei physiologischen Experimenten dieser Art nicht bemerkbar machen. Anders aber steht es mit dem vorderen Brennpunkt, der seine Lage recht merkbar ändert - in dem betrachteten Bereich um nahezu $4 \mathrm{~mm}$ - und zwar verschiebt er sich mit wachsender Ametropie für Hyperopen nach hinten, für Myopen nach vorn, beidemal im Sinne der Lichtrichtung gemessen.

Die starke Durchbiegung der beiden Linsenflächen hat auch zur Folge, dass ein afokales Kontaktglas, wie es für emmetropische Augen in diesem Schema zur Aufrechterhaltung der Kontinuität zu konstruieren ist, das Netzhautbild nicht ungeändert lässt. Der Vorderradius $r_{1}$ dieses afokalen Systems ergibt sich aus dem Hinterradius $r_{2}$, der Dicke $d$ und dem Brechungsexponenten $n$ nach der an anderm Orte abgeleiteten Formel zu

$$
r_{1}=r_{2}+\frac{n-1}{n} d,
$$

und das so gebildete System wirkt nach Art eines duinnen Steinheilschen Conus, mit der Vergrösserungsziffer

$$
V=1,02 \text {. }
$$

Nach der graphischen Darstellung ändert eine korrigierende Ficksche Kontaktbrille mit der Dicke von $0,4 \mathrm{~mm}$ nur dann die Grösse des Netzhautbildes nicht, wenn sie eine Brechkraft von

$$
D_{1}=1 \mathrm{dptr}
$$

hat.

In allen andern Fällen ist, wie schon gesagt, eine Veränderung der Grösse des Netzhautbildes mit der Anwendung dieses Korrektionsmittels verbunden, und es wird sich aus späteren Teilen dieser Arbeit ergeben, dass die Kenntnis dieser Veränderung notwendig ist, wenn gewisse, ziemlich naheliegende Fehler vermieden werden sollen.

Im allgemeinen gesprochen ergeben sich kleinere Netzhautbilder, ergibt sich also eine geringere Sehschärfe für alle $1 \mathrm{dptr}$ übersteigenden Grade der Hyperopie, und grössere Netzhautbilder, also eine höhere Sehschärfe für alle die übrigen axialen Refraktionen. Die relative Sehschärfe $S_{r}$ lässt sich nach der Gullstrandschen Formel bestimmen $\mathrm{zu}$ 


$$
\begin{aligned}
S_{r^{\prime}} & =S\left(1+\delta_{f} L\right) \\
& =S\left(1+\frac{H^{\prime} \mathbf{F}}{\mathbf{F} O^{\prime}}\right)=S\left(1+\frac{H O^{\prime}+O^{\prime} \mathbf{W}}{\mathbf{F} O^{\prime}}\right) \\
& =S\left(1+\frac{H O^{\prime}}{\mathbf{F} O^{\prime}}-1\right) \\
& =S \frac{H^{\prime} O^{\prime}}{\mathbf{F} O^{\prime}}
\end{aligned}
$$

sie wird kleiner als die absolute für die stärkeren Sammelgläser und grösser als die absolute Sehschärfe für die schwächsten Sammel- und fiir alle Zerstreuungsgläser.

Hier also liegt wieder ein Fall vor, wo man von einem Vorteil sprechen kann, den die Achsenverlängerung des myopischen Auges bietet, obwohl sie zweifellos im allgemeinen ein Nachteil ist. Einer von uns $\left.{ }^{1}\right)$ hatte bei einer bestimmten Art der Fernrohrbrille darauf hingewiesen, dass der Myop innerhalb eines freilich beschränkten Blickfeldes aus der anomalen Achsenlänge einen Vorteil ziehen könne. Hier aber fällt sogar die Einschränkung des Blickfeldes fort, und ein Myop von normaler Sehschärfe $S$ erhält - theoretisch wenigstens infolge der Achsenverlängerung seines Auges durch die Korrektion mit der Kontaktbrille eine höhere Sehschärfe als ein Emmetrop mit derselben normalen Sehschärfe $S$.

Prinzipiell in gleicher Weise zu behandeln sind die Fälle, in denen die Kontaktbrille angewandt wird, um eine bestimmte Ametropie herbeizuführen. Aus den Anfangswerten, meistens aus dem für das neue krïmmungsametropische Auge vorgeschriebenen Fermpunktsabstande, lässt sich die Brechkraft des vorzuschaltenden Kontaktglases ermitteln, und die Abbildungskonstanten sind für das kombinierte System mit Hilfe derselben Eormeln aus der Dioptrienrechnung zu erhalten, die für die Bestimmung der oben gegebenen Figur gedient haben. Einige nähere Angaben über die Einzelfälle werden im nachstehenden noch gemacht werden.

Sind auf diese Weise die optischen Konstanten des mit einer Eickschen Kontaktbrille bewaffneten Auges ermittelt worden, so kann man nun dazu übergehen, die in dem Titel versprochenen subjektiven Prifungsmethoden und die von ihnen gelieferten Resultate zu beschreiben.

1) v. Rohr, M., Zur Theorie der Fernrohrbrille, r. Graefe's Arch. f. Ophth. Bd. LXXV. S. $561-585$. 1910. Siehe S. 581. 


\section{Die Prüfung der Korrektionsmöglichkeit der Anisometropie.}

Für das beidäugige Sehen Anisometroper gibt es noch keine allgemein angenommene Theorie, und man braucht nur in die modernste Darstellung dieses Gebietes ${ }^{1}$ ) Einblick zu nehmen, um zu sehen, dass sowohl Fälle beschrieben sind, wo bei geringem Betrage der Anisometropie das beidäugige Sehen ausblieb, als auch solche, wo es zu stande kam, obwohl es sich um einseitige Aphakie handelte. Von besonderer Bedeutung erschien uns in der citierten Darstellung die von C. Hess ausgesprochene Ansicht, dass es sich beim beidäugigen Sehen um deutliche Abstufungen handeln könne, und wir beben schon hier hervor, dass wir diese Ansicht bei unsern Versuchen bestätigt gefunden haben. Eben darum, weil die Ansichten über dieses Problem des beidäugigen Sehens noch nicht allgemein befestigt waren, schienen uns einige Versuche mit den Kontaktbrillen nicht ohne Interesse zu sein, weil es sich hier um Experimente handelte, die einerseits leicht wiederholt werden und die anderseits von geschulten Beobachtern mit normalem Muskelapparat angestellt werden konnten.

Es wurde zunächst das rechte Auge M. v. Rohrs $(A=-6,4$ dptr) durch Aufsetzen einer Fickschen Kontaktbrille emmetropisch gemacht. Wegen der Schwierigkeit der Herstellung beschränkten wir uns bei unsern Versuchen mit dem optisch wirksamen Teil der Kontaktbrille, der Glascornea in der Fickschen Ausdrucksweise. Wir gingen beim Aufsetzen so ror, dass der auf einem Stuhle sitzende Beobachter bei horizontaler Haltung des Kopfes das zu bewaffnende Auge nach der Einträufelung von Kokain direkt nach unten richtete, während er sein Unterlid herabzog. Alsdann füllte man das Kontaktglas mit physiologischer Kochsalzlösung und drückte es dem Beobachter von unten her an die Cornea des senkrecht hinabblickenden Auges an. Es haftete meistens gleich gut, d. h. ohne Luftblasen, und es bedurfte nur geringer Verschiebungen, um einen zentrierten Sitz herbeizuführen. Der Lidschlag bewegte das Glas manchmal ein wenig, so dass bei den ersten Versuchen nicht immer das Auftreten von Astigmatismus vermieden worden sein wird.

Wie wir bereits oben auseinandergesetzt haben, führt man durch das Kontaktglas, dessen richtiger Sitz vorausgesetzt sei, eine gewisse

1) Hess, C., Die Refraltion und Accommodation des menschlichen Auges und ihre Anomalien. Graefe-Saemisch-Hesssches Handb. d, ges, Augenheilk. 3. Aufl. von 1908-1910. S. 590-599. 
Krümmungsametropie ein, und das Netzhautbild erhält in dem vorliegenden Falle eine Vergrösserung

$$
V=1,14 \text {. }
$$

Es war dem Beobachter, der auf dem korrigierten Auge eine etwas herabgeminderte Sehschärfe hatte, nicht möglich, bei der bestehenden Anisometropie von mehr als $6 \mathrm{dptr}$ die beiden Bilder zu vereinigen. Dies gelang weder bei fernen noch bei nahen Objekten. Der Eindruck des Sehens mit beiden Augen war unter diesen Umständen in hohem Masse peinlich.

Ess lag somit der Gedanke nahe, das andere ametropische Auge durch Bewaffnung mit einem gewöhnlichen Brillenglase zu korrigieren. Dabei kann man theoretisch sicherlich die gleiche Bildgrösse auf der Netzhaut erhalten, wie sie bei einem emmetropischen Auge der gleichen Brechkraft auftreten würde. Es ist dazu janurnötig, das korrigierende Brillenglas so anzubringen, dass sein hinterer Hauptpunkt $H^{\prime}$ mit dem objektseitigen Brennpunkt $\mathbf{F}$ des accommodationslosen ametropischen Auges zusammenfällt. Aber auch weun das Brillenglas nicht ge-

Fig. 4. Schematische Darstellung der Änderung der Blickwinkel $w, w^{\prime}$ durch ein korrigierendes Brillenglas. nau den theoretisch richtigen Ort einnimmt, sondern um mehrere Millimeter von ihm abweicht, tritt an dem Netzhautbild keine wesentliche Grössenänderung auf, wie eine einfache Rechnung sofort zeigt, wenn man dem Interstitium $\delta$ etwa die beiden Werte 17,06 und $13,3 \mathrm{~mm}$ beilegt.

Nicht so einfach verhält es sich aber mit der Änderung der Blickwinkel. Die Einfübrung eines korrigierenden Brillenglases in endlicher Entfernung von dem bewegten Auge führt in folgender Weise einen Unterschied zwischen objektseitigen und augenseitigen Blickwinkeln herbei. Es sei in Fig. 4 ein zerstreuendes Brillenglas $L$ vorausgesetzt, so bildet es den (um $S Z^{\prime}=25 \mathrm{~mm}$ entfernten) dem Augenraum angehörenden Augendrehpunkt $Z^{\prime}$ im Objektraum in $Z$, dem scheinbaren Augendrehpunkt, ab, und es sind nummehr diese beiden Drehpunkte für den Objekt- und den Augenraum zu unterscheiden. Von der sphärischen Aberration, mit der $Z$ im allgemeinen behaftet sein wird, sei einmal abgesehen, dann dienen die beiden Punkte $Z$ und $Z^{\prime}$ als Scheitel je für die objektseitigen und die augenseitigen Blickwinkel $w$ und $w^{\prime}$. Wie man aus dem Betrage der Abstandsdifferenz $Z Z^{\prime}$ schliessen kann, nimmt der Unterschied $w-w^{\prime}$, 
Über eine Methode zur subjektiven Prüfung von Brillenwirkungen. 199

die prismatische Ablenkung, bei Brillengläsern von mittlerer oder böherer Brechkraft sehr merkliche Werte an, was sich in folgender Weise beim Blicken störend bemerklich machen kann.

Im Objektraum ergeben sich für das mit dem Fickschen Kontaktglas und für das mit dem korrigierenden Brillenglas gewöhnlicher Art versehene Auge sicherlich verschieden gelagerte Drehpunkte. Denn während das mit dem Kontaktglas ausgerüstete Auge seinen ursprünglichen Drehpunkt beibehält, erfährt nach dem vorhergehenden der entsprechende Punkt des andern eine Lagenänderung nach $Z_{b}$, so dass die Lage der verschiedenen Punkte die folgende sein mag (Fig. 5). Bei einigermassen grossen Objektabständen indessen bedeutet die Verlagerung bei dem mit der gewöhnlichen Brille bewaffneten Auge wenig, und man kann für die praktischen Verhältnisse die Drehung der Augenbasis (von $Z_{r} Z_{l}^{\prime}$ nach $Z_{r} Z_{l}$ ) unbeachtet lassen und annehmen, dass die objektseitigen Blickwinkel des linken Auges dieselbe Grösse haben, die ihnen zukommen würde, wenn das Auge nicht bewaffnet wäre. In dem Augenraum aber gelten für das rechte, mit der Kontaktbrille ausgerüstete Auge die ungeänderten Blickwinkel, während sie für das linke durch die merklich verschiedenen augenseitigen $w^{\prime}$ zu ersetzen sind.

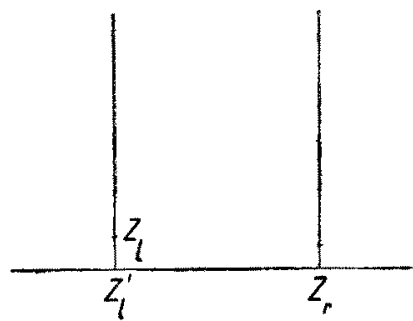

Fig. 5. Die Verlagerung der Augenbasis im Objektraum. Schematische Darstellung im Grundriss.

Bewaffnete der Beobachter sein linkes Auge mit der korrigierenden Brille in einem Abstande von

$$
\partial=12 \mathrm{~mm}
$$

zwischen dem inneren Brillen- und dem Hornhautscheitel, so war die Vereinigung der Bilder in der Mitte des Feldes möglich, doch lagen die Bilder in den Seitenteilen des Blickfeldes getrennt. Richtete er seine Aufmerksamkeit auf solche seitlich gelegenen Objekte, so traten die in der Mitte des Blickfeldes liegenden Gegenstände auseinander.

Wir sind der Meinung, dass diese Erscheinung auf die merkliche Verschiedenheit zurückzuführen ist, die, wie soeben auseinandergesetzt wurde, infolge der Wirkung des korrigierenden Brillenglases zwischen den Drehungswinkeln des linken und des rechten Auges besteht. Wie gross die Unterschiede werden können, zeigt die folgende kleine Zusammenstellung. 
Der Zusammenhang zwischen objektseitigen $(u)$ and augenseitigen $\left(w^{\prime}\right)$ Blickwinkeln für ein punktuell abbildendes Brillenglas $A_{\infty}=-7 \mathrm{dptr}:$

$$
\begin{array}{lll}
w^{\prime}=0^{\circ} & 20,7^{\circ} & 30^{\circ} \\
w=0^{\circ} & 24,6^{\circ} & 36^{\circ} .
\end{array}
$$

Fiir alle Punkte ausserhalb der durch die Augenbasis gelegten Horizontalebene wird dadurch - neben einem gewissen, hier nicht weiter behandelten Konvergenzwinkel - ein Höhenfehler eingeführt, der zwar beim Fixieren seitlicher Objekte überwunden wurde, der sich aber doch bei der Orientierung im Blickfelde deutlich bemerkbar machte.

Binokulares Sehen und beidäugige. Tiefenwahrnehmung waren nicht unmöglich, aber doch mit einer peinlichen Anstrengung verbunden, und die Exaktheit der Tiefenangabe war wesentlich herabgesetzt. An dem Hegnerschen Testobjekt wurden die Nägel ${ }^{1}$ ) wohl getroffen, aber das geschah doch erst nach einigen Versuchen und mit wesentlich grösseren als den unter normalen Verhältnissen gemachten Fehlern.

Im ganzen liess sich aus diesen Versuchen, die mehrere Male, etwa je eine halbe Stunde hindurch, fortgesetzt wurden, der Schluss zaiehen, dass die Herabsetzung der ursprünglichen, sehr guten beidångigen Tiefenwahrnehmung durch die Herbeiführung einer Anisometropie höheren Grades ganz ausserordentlich merkbar war, und dass sie auch dann nicht aufgehoben wurde, wemn das ametropische Auge durch eine Brille gewöhnlicher Art korrigiert wurde. Der Beobachter hätte den gewohnten Gebrauch seiner Augen verlernen und sich einen neuen erwerben müssen, wenn ihm eine äbnliche Sicherheit im beidäugigen Sehen wie vorher hätte erreichbar werden sollen.

Bewaffnete M. v. Rohr dagegen sein linkes Auge mit einer besonderen, von ihm früher konstruierten A nisometropbrille mit

$$
A_{\infty}=-7 \mathrm{dptr}
$$

so fielen die Versuche wesentlich anders aus.

Bevor diese beschrieben werden, sollen einige Angaben über das System gemacht werden. Ein Patient hatte sich in der Jenaer Augenklinik eingestellt, der bei voller Sehschärfe auf beiden Augen für das eine ein Korrektionsglas von -7 dptr bedurfte, während das anm

1) Hegner, O. A., Über oin noues Brillensystem gur Korrektion einseitiger Aphakie. Klin. Monatsbl. f. Augenheilk. Bd. XII. S. 273-278. + 1912 Märzheft). 
Über eine Methode zur subjektiven Prüfung von Brillenwirkungen. 201

dere Auge emmetropisch war. Da der Patient auf Befragen angegeben batte, er empfände das Bild des durch die Brille korrigierten Auges etwas kleiner als das des emmetropischen Auges, so war die Berechnung für ein in Figur 6 dargestelltes Fernrohrbrillensystem gemacht worden, das eine Vergrösserung von etwa $10 \%$ os genauer

$$
V_{k}=1,12
$$

herbeiführte. Die weiteren Daten waren so gewählt worden, dass sich für das Blickfeld von $50^{\circ}$ eine punktuelle Abbildung ergab, und dass Verzeichnung und merkbare Farbenabweichung vermieden waren. Die augenseitigen Drehungswinkel waren von den objektseitigen nur wenig verschieden und zwar fielen die letztgenannten etwas grösser aus

$$
\begin{array}{lll}
w^{\circ}=0^{\circ} & 17,39^{\circ} & 25^{\circ} \\
w=0^{\circ} & 18,66^{\circ} & 26,69^{\circ} .
\end{array}
$$

Es sei gleich hier bemerkt, dass der Patient nicht die Geduld

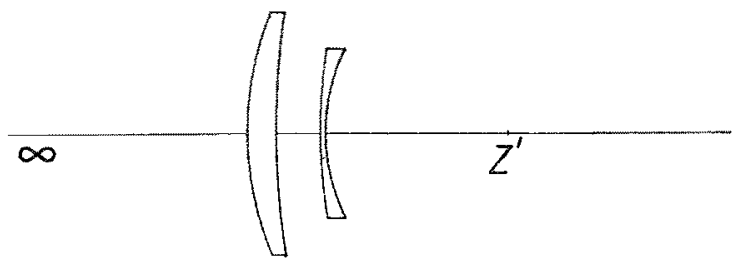

Fig. 6. Konstruktionsschema für eine punktuell abbildende Anisometropbrille $A_{\infty}=-7$ dptr.

hatte, die Übungen am. Stereoskop durchzuführen, obwohl sie einen Erfolg erwarten liessen. Wir haben aus seinen Aussagen nicht mehr entnehmen können als ein non liquet.

Man erkennt, dass unter diesen Umständen M. v. Rohr dann, wenn er links die Anisometropbrille, rechts die Kontaktbrille benutzte, auf beiden Augen korrigiert war und Netzhautbilder nahezu gleicher Vergrösserung erhielt, während die Drehungswinkel seiner beiden Augen zwar nicht völlig aber doch angenähert übereinstimmten. Der Erfolg mit der Anisometropbrille war sehr befriedigend: die beiden Bilder verschmolzen unmittelbar, und die stereoskopische Wahrnehmung erfolgte mit der gleichen Sicherheit wie bei der Korrektion durch die gewohnte, dauernd getragene Brille.

Es will uns scheinen, dass man aus diesem Versuchsergebnis den folgenden Schluss ziehen könne. War wirklich das Netzhautbild des myopischen Auges unseres Patienten um etwa $12 \%$ zu klein, wemn die Korrektion durch ein dünnes Brillenglas ungefähr am vorderen Augenbrennpunkte herbeigeführt wurde, so ist anzunehmen, dass mit 
dem Anisometropglas dieser Patient gut beidäugig würde haben sehen können, wenn es ihm gelungen wäre, seine Schielstellung zu überwinden. Denn einmal wäre durch das Anisometropglas das zu kleine Netzhautbild auf die gleiche Grösse mit dem des andern Auges gebracht worden, und ferner hätten nur geringe Grössenunterschiede der Blickwinkel bei beideu Augen bestanden.

In einem gewissen Zusammenhange mit diesem Experiment steht ein Versuch mit einem einseitig-aphakischen Patienten. Die bereits citierte Hegnersche Mitteilung behandelte mehrere Fälle, in denen eine einseitige traumatische Katarakt erfolgreich operiert worden war. Die Patienten konnten mit den gewohnten Stargläsern korrigiert keine beidäugige Tiefenwahrnehmung erhalten, während ibnen eine solche in recht befriedigender Weise durch ein besonderes korrigierendes Starglas vermittelt wurde. Die Konstruktion musste aus zwei voneinander getreunten Systemen von verschiedenem Vorzeichen der Brennweite zusammengesetzt werden und zwar derart, dass das zerstreuende System den Objekten, das sammelnde dem Auge zugekehrt war.

Fiur die Berechnung eines derartigen Glases für Einseitig-Aphakische musste ausser der Bestimmung der Refraktion und der Brechkraft des Hornhautsystems auch noch eine Angabe über die Brechkraft gemacht werden, die dem brillenbewaffneten aphakischen Auge durch die Korroktion verliehen werden sollte. Sie ergab sich ohne Zwang aus der Forderung gleicher Netzhautbildgrösse wie in dem gesunden Auge. Unter diesen Umständen sind für das vorzuschaltende System wichtige Konstanten der Gaussschen Abbildung bestimmt, nämlich die Brechkraft und der Abstand seines hinteren Hauptpunktes von dem objektseitigen Augenhauptpunkt. Was noch wählbar bleibt, ist etwa der Luftabstand zwischen der negativen und der positiven Komponente, den man aus verständlichen Grïnden nicht über eine gewisse, an sich geringe Grösse hinauswachsen lassen kann, wenn das Starglas für Einseitig-Aphakische nicht unnötig auffällig werden soll.

Nimmt man noch die gleichsam stillschweigend bestehenden Bedingungen hinzu, dass das System für ein Blickfeld endlicher Ausdehnung punktuell abbilde, verzeichnungsfrei sei und keine störenden Farbenabweichungen zeige, so bestimmen sich die Linsenformen im Verlauf der Rechnung. Es zeigt sich dabei, dass das Sammelglied in zwei Bestandteile zerlegt werden muss, wenn man die punktuelle Abbildung allein mit sphärischen Flächen erreichen will. 
Über eine Methode zur subjektiven Prüfung von Brillenwirkungen. 203

Für ein aphakisches Auge mit normaler Hornhautkrümmung und eine korrigierende Brille von

$$
A_{\infty}=13 \mathrm{dptr}
$$

ergibt sich das in der untenstehenden Fig. 7 dargestellte System, und zwar ist es so beschaffen, dass die beim Blicken auftretenden Winkel $w$ und $w^{\prime}$ die folgenden Beziehungen zueinander haben:

$$
\begin{array}{lll}
w^{\prime}=0^{\circ} & 17,388^{\circ} & 25,0^{\circ} \\
w=0^{\circ} & 14,187^{\circ} & 20,7^{\circ} .
\end{array}
$$

Das sieht nun zunächst nicht so aus, als ob eine besonders gute Übereinstimmung zwischen den objektseitigen und den augenseitigen Blickrichtungen erreicht worden wäre, aber man erkennt den Fortschritt sofort, wenn man diese Werte mit denen vergleicht, die sich

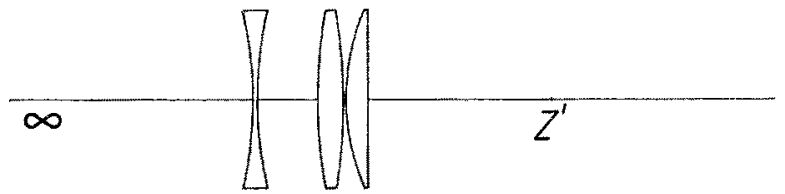

Fig. 7. Konstruktionsschema für ein punktuell abbildendes Brillenglas von $A_{\infty}=13$ dptr für Einseitig-Aphakische.

bei einem Gullstrandschen Starglas gleicher Brillenscheitelrefraktion ergeben; für die gleichen $w^{\prime}$-Winkel ergibt sich

$$
w=0^{\circ} \quad 11,5^{\circ} \quad 15,8^{\circ}
$$

und man erkennt daraus, dass die Verminderung der prismatischen Ablenkung recht merkbar ist.

An und für sich würde es möglich sein, die Grösse der augenseitigen Blickwinkel den objektseitigen noch mehr anzunähern, doch wï̈rde man dann gegen die Forderung gleicher Netzhautbildgrösse verstossen, während für diese Versuche daran stets festgehalten wurde.

Schon C. A. Hegner hat in seinem auf S. 12 angeführten Artikel über Patienten berichtet, die mit den neuen Brillen für Einseitig-A phakische gute Resultate hatten, und auch von einem andern Ophthalmologen ${ }^{1}$ ) sind uns solche günstigen Ergebnisse berichtet worden. Die Patienten gewöhnten sich sehr rasch an eine solche Brille, wobei es vorderhand unentschieden bleibt, ob die hier bevorzugte Konstanz der Grösse des Netzhautbildes oder die geringere Abweichung

1) Wolff, H., Korrektion der Anisometropie bei Aphakie mittels eines Z eiss schen zweigliedrigen Linsensystems. Zeitschr. f. Augenheilk. Bd. XXVIII. S. 149-150. 1912. (Zusatz bei der Korrektur.) 
der Richtungen beider Blicklinien fuir den befriedigenden Ausfall der Versuche verantwortlich zu machen ist.

Das Ficksche Kontaktglas kann möglicherweise anch hier zu der Entscheidung beitragen, doch haben wir den Versuch zunächst nur so angelegt, dass es sich um die Konstanterhaltung der Netzhautbildgrösse handelte. Macht man nämlich durch ein Kontaktglas der oben angegebenen Form ein Gullstrandsches schematisches Auge künstlich aphakisch, indem es auf einen virtuellen Objektpunkt eingestellt wird, der $65,37 \mathrm{~mm}$ von dem Hornhantscheitel entfernt 1st, so wird seine Fovea durch die Vorschaltung des oben beschriebenen Systems für Einseitig-Aphakische dem unendlich fernen Objektpunkt konjugiert. Dabei bleibt aber die Grösse des Netzhautbildes unverändert.

Da wir beide unserer myopischen Augen wegen nicht in Betracht kamen, so hatte Herr Dr. Erggelet, ein Assistent der Jenaer Augenklinik, die Freundlichkeit, diese Versuche für uns anzustellen. Er ist Emmetrop mit achsensymmetrischer Hornhant und hoher Sehschärfe. Die Versuche waren insofern unbequem, als die ihm aufgesteckte Kontaktbrille eine stark zerstreuende Wirkung haben musste und mithin am Rande merklich stärker war als die unsrigen. Es war daher notwendig, sie häufiger zu richten, da sie durch den Lidschlag leicht verschoben wurde.

Die Versuche, denen sich Herr Frggelet in dankenswerter Bereitwilligkeit unterzog, führten zu den folgenden Ergebnissen.

Korrigierte er sein aphakisch gemachtes Auge mit einem eintachen Brillenglase möglichst geringer Dicke, so war der Grössenunterschied der beiden Bilder auffällig; und die seitlichen Objekte wurden von den beiden Augen an verschiedenen Stellen wahrgenommen, wenn er schief durch das korrigierende Brillenglas blickte. Dem gegenüber erschienen bei der Verwendung des Systems für Einseitig-Aphakische die Bilder in beiden Augen gleich gross, und die Winkelverschiedenheit bei schiefem Blick durch das System war deutlich geringer. Allerdings gelang es bei den Versuchen nicht, die Doppelbilder am Rande des Blickfeldes zu vereinigen. Eine beidaugige Tiefenwahrnehmung war aber entschieden vorhanden, wenn man nicht gar zu weit aus der Mitte des Blickfeldes hinausging.

Zieht man aus diesen Versuchen die Folgerungen, so weichen sie namentlich darin von den Angaben der ungeschulten EinseitigAphakischen ab, dass die Vereinigung der Bilder beim seitlichen Blick durch das System dem Ophthalmologen nicht gelang. Man kann 
Über eine Methode zur subjektiven Prüfung ron Brillenwirkungen. 205

aber wohl darauf hinweisen, dass bei den Erggeletschen Versuchen, die immer nur einige Minuten währten, die Übung und Gewöbnung im Gegensatz zu der Lage bei den Patienten keine Rolle spielen konnte. Nach der Untersuchung von C. A. Hegner ${ }^{1}$ ) kann man aber annehmen, dass diese Übung hier eine grosse Rolle spielen wird, weil die vorliegenden Richtungsverschiedenheiten von derselben Grössenordnung sind, die C. A. Hegner sogar bei einem reinen Unterschiede der Erhebungswinkel zu überwinden lernte.

Mithin sprechen auch in diesem Falle die Resultate der Prüfung, die durch die Ficksche Kontaktbrille einem physiologisch-psychologisch geschulten Ophthalmologen ermöglicht wurde, nicht gegen die Gedanken, die den Experimenten zugrunde lagen. Natürlich bleibt es die Frage, ob nicht anch dann ein Weg zur Lösung des Problems führe, wenn man von der Forderung gleicher Netzhautbildgrösse abginge zugunsten einer besseren Übereinstimmung von bild- und objektseitigem Blickwinkel. Leider muss die Behandlung dieser Aufgabe vorläufig zurückgestellt werden, doch wird man diese Möglichkeit im Auge behalten müssen.

Was aber der praktischen Bedeutung aller Bestrebungen, den Einseitig-Aphakischen zu helfen, ein ungünstiges Prognostikon stellt, ist die weit verbreitete Rücksicht auf die Schönheit des Aussehens. Die Annahme, es würde eine Vorrichtung, die dem Einseitig-Aphakischen eine wirklich befriedigende binokulare Tiefenwahrnehmung wiedergäbe und damit die Operation richtig auszunutzen gestatte, mit Freude begrüsst werden, erwies sich als kindlich und weltfremd. So merkwürdig es klingt, richtet sich die Hauptforderung des Patienten weniger auf die Verwendung des glïcklich operierten Auges, als vielmehr auf die möglichste Vermeidung eines auffälligen Aussehens, oder unter Benutzung eines Fraunhoferschen Ausspruchs, der Patient wünscht die Brille mehr zum Ansehen als zum Durchsehen. Da aber bedauerlicherweise die optischen Gesetze tyrannisch bei einem System für Einseitig-Aphakische einen endlichen Iinsenabstand fordern, was also eine immerhin auffallende Brillenform zur Folge hat, so hat es houte den Anschein, als würde diese Konstruktion verhältnismässig selten getragen werden.

1) Hegner, C. A., Zur Verteilung der überwindbaren Höhenfehler im Blickfelde. Habilitationsschr. Jena 1912. 48 S. $8^{\circ} .13$ t. Siehe S. 34 . 